\title{
Resveratrol Ameliorates Arterial Stiffness Assessed by Cardio-Ankle Vascular Index in Patients With Type 2 Diabetes Mellitus
}

\author{
Haruki Imamura, ${ }^{1}$ MD, Takashi Yamaguchi, ${ }^{1}$ MD, Daiji Nagayama, ${ }^{1}$ MD, \\ Atsuhito SaIKI, ${ }^{1} \mathrm{MD}$, Kohji ShIRAI, ${ }^{1} \mathrm{MD}$, and Ichiro Tatsuno, ${ }^{1} \mathrm{MD}$
}

\begin{abstract}
SUMMARY
Resveratrol has been reported to have potent anti-atherosclerotic effects in animal studies. However, there are few interventional studies in human patients with atherosclerogenic diseases. The cardio-ankle vascular index (CAVI) reflects arterial stiffness and is a clinical surrogate marker of atherosclerosis. The aim of the present study was to investigate the effect of resveratrol on arterial stiffness assessed by CAVI in patients with type 2 diabetes mellitus (T2DM).

In this double-blind, randomized, placebo-controlled study, 50 patients with T2DM received supplement of a 100$\mathrm{mg}$ resveratrol tablet (total resveratrol: oligo-stilbene $27.97 \mathrm{mg} / 100 \mathrm{mg} /$ day) or placebo daily for 12 weeks. CAVI was assessed at baseline and the end of study. Body weight (BW), blood pressure (BP), glucose and lipid metabolic parameters, and diacron-reactive oxygen metabolites (d-ROMs; an oxidative stress marker) were also measured.

Resveratrol supplementation decreased systolic BP $(-5.5 \pm 13.0 \mathrm{mmHg}), \mathrm{d}-\mathrm{ROMs}(-25.6 \pm 41.8 \mathrm{U} . \mathrm{CARR})$, and CAVI $(-0.4 \pm 0.7)$ significantly $(P<0.05)$ and decreased BW $(-0.8 \pm 2.1 \mathrm{~kg}, P=0.083)$ and body mass index $(-0.5 \pm 0.8$ $\mathrm{kg} / \mathrm{m}^{2}, P=0.092$ ) slightly compared to baseline, while there were no significant changes in the placebo group. Decreases in CAVI and d-ROMs were significantly greater in the resveratrol group than in the placebo group. Multivariate logistic regression analysis identified resveratrol supplementation as an independent predictor for a CAVI decrease of more than 0.5 .

In conclusion, 12-week resveratrol supplementation may improve arterial stiffness and reduce oxidative stress in patients with T2DM. Resveratrol may be beneficial in preventing the development of atherosclerosis induced by diabetes. However, a large-scale cohort study is required to validate the present findings. (Int Heart J 2017; 58: 577-583)
\end{abstract}

Key words: Polyphenol, Atherosclerosis, Vascular function, Oxidative stress, d-ROMs, Weight loss, Body weight

I type 2 diabetes mellitus (T2DM), hyperglycemia, insulin resistance, and dyslipidemia are well known to induce atherosclerosis. ${ }^{1-3)}$ Although the current incidence of atherosclerotic diseases in Japan is relatively low compared to that in other countries, an increase is expected in the near future with the increase of diabetic patients. Therefore, treatment for atherosclerosis in diabetic patients is an important concern.

Resveratrol, a polyphenol present in grapes and red wine, is known to have potent lifespan extension effects through activation of Sirt1, a NAD-dependent deacetylase. ${ }^{4,5)}$ Interestingly, resveratrol also exhibits an anti-atherosclerotic effect, although the evidence is limited to animal experiments. ${ }^{6,7)}$ Therefore, resveratrol has attracted attention as a candidate for antiatherosclerosis therapy. ${ }^{8,9)}$

Recently, a novel arterial stiffness diagnostic parameter called the cardio-ankle vascular index (CAVI) has been developed in Japan, which essentially reflects the stiffness of the aorta, femoral artery, and tibial artery. ${ }^{10,11)}$ This stiffness parameter has been reported to be independent of blood pressure and to have adequate reproducibility for clinical use. ${ }^{12)}$ Furthermore, no special technique is required for the measurement of CAVI. Indeed, CAVI is associated with a number of coronary risk factors and the severity of cardiovascular disease. ${ }^{13)} \mathrm{Al}-$ though resveratrol is considered to have potent anti-atherosclerotic effects in experimental animals, there are few reports on studies in human patients. On the other hand, resveratrol is known to affect oxidative stress, body adiposity, and insulin sensitivity, which are all involved in the development of atherosclerosis.

In this study, we examined the effects of resveratrol on arterial stiffness assessed by CAVI and on its underlying mechanisms in patients with T2DM.

\section{Methods}

This study protocol was prepared in accordance with the Declaration of Helsinki and was approved by the institutional

From the ${ }^{1}$ Center of Diabetes, Endocrine and Metabolism, Toho University Sakura Medical Center, Chiba, Japan.

Address for correspondence: Ichiro Tatsuno, MD, Center of Diabetes, Endocrine and Metabolism, Toho University Sakura Medical Center, 564-1 Shimoshizu, Sakura, Chiba 285-8741, Japan. E-mail: ichiro.tatsuno@med.toho-u.ac.jp

Received for publication August 8, 2016. Revised and accepted November 2, 2016

Released in advance online on J-STAGE July 13, 2017.

All rights reserved by the International Heart Journal Association. 
review board of Sakura Hospital, Toho University Medical Center (approval number 2011-074). Before participation, we explained the purpose of the study to each subject, and consent was obtained for participation in the study and for release of the study data.

Patient recruitment: This study was conducted as a doubleblind placebo-controlled randomized clinical trial. Patients who met the inclusion criteria were invited to take part in the study. At the first visit, the benefits of participation were explained and they were informed of the research goals, method of intervention, and duration of the study. Among 60 patients who were interviewed, 50 eligible patients with T2DM (HbA1c $>7.0 \%)$ were recruited for the study. The subjects received supplement of a 100-mg resveratrol (total resveratrol: oligo-stilbene $27.97 \mathrm{mg} / 100 \mathrm{mg} /$ day) or placebo tablet for 12 weeks (study period: April 2012 to April 2014). Before beginning, the bottles containing capsules for both groups were marked by an individual other than the researchers with $\mathrm{A}$ and $B$ codes in order to keep the researchers and patients uninformed about the type of each group (considering the doubleblind characteristic of the study). Resveratrol was obtained from BHN Corporation (Tokyo) as resveratrol- $\varepsilon$ which is derived from grape stems. Patients were excluded if they had severe hyperglycemia $(\mathrm{HbA} 1 \mathrm{c}>9.0 \%)$; an allergy to shrimp and/or crab; were pregnant or breast-feeding; or had a history of cerebrovascular disease, other vascular diseases, definitive renal disease, or severe liver dysfunction. Patients with anklebrachial indices lower than 0.90 and atrial fibrillation were also excluded because CAVI was apparently low in patients with severe arterial occlusive diseases. ${ }^{13)}$

Data collection: The following parameters were measured before and after 12 weeks of intervention: body weight (BW), body mass index (BMI), fasting plasma glucose (FPG), HbA1c, general blood tests (total protein, albumin, aspartate aminotransferase, alanine aminotransferase), serum total cholesterol (T-C), triglycerides (TG), high-density lipoprotein cholesterol (HDL-C), low-density lipoprotein cholesterol (LDL-C), and diacron-reactive oxygen metabolites (d-ROMs) as a oxidative stress marker. Systolic blood pressure (BP), diastolic BP, and CAVI were also measured before and after 12 weeks. Blood samples were collected after overnight fasting and patients with a triglyceride level exceeding $400 \mathrm{mg} / \mathrm{dL}$ were excluded. All patients maintained the same diet and exercise therapies. They were also asked to inform the researcher of any changes made in the type or dosage of the drugs and did not change medications during this study. A dietician provided nutritional guidance to all subjects on a monthly basis, analyzing meals and suggesting changes if necessary. The primary outcome was the changes of CAVI after treatment by resveratrol versus placebo supplementation. The secondary outcomes were the changes in the following parameters after treatment by resveratrol versus placebo supplementation: systolic BP, diastolic BP, BW, BMI, d-ROMs, FPG, HbA1c, T-C, TG, HDL$\mathrm{C}$, and LDL-C.

\section{Assay methods:}

HbAlc and plasma lipid concentrations To measure HbA1c, blood was collected into tubes containing ethylenediaminetetraacetic acid. The stable and unstable fractions of HbA1c were measured by a high-pressure liquid chromatography method (Hi-Auto A1c Analyzer System; Kyoto Daiichi Kagaku, Kyoto, Japan). Data for the stable form were used in the present analysis. HbA1c was expressed as the value of the National Glycohemoglobin Standardization Program (NGSP).

Plasma total cholesterol and triglyceride levels were measured enzymatically using commercial kits (Nippon Shoji Co, Ltd., Osaka, Japan) and a Hitachi 7150 Autoanalyzer (Hitachi, Ltd., Tokyo). Serum high-density lipoprotein cholesterol was measured using a selective inhibition assay (Daiichi Pure Chemicals Co, Ltd, Tokyo). Serum LDL-cholesterol levels were calculated using the Friedewald formula.

Measurements of CAVI and blood pressure The CAVI was measured using a VaSera CAVI instrument (Fukuda Denshi Co. Ltd., Tokyo) by a method described previously. ${ }^{10)}$ Briefly, cuffs were applied to the bilateral upper arms and ankles, with the subject lying supine and the head held in a midline position. After resting for 10 minutes, the examinations were performed. To detect the brachial and ankle pulse waves with cuffs, a low cuff pressure from 30 to $50 \mathrm{mmHg}$ was used to minimize the effect of cuff pressure on hemodynamics.

Resting systolic and diastolic blood pressures were taken after the participants spent 10 minutes seated in a quiet room, free of distractions. Blood pressure was obtained according to a standardized protocol (Chobanian, et al, 2003) by a registered nurse involved in the study. Blood pressure was taken from the brachial artery through auscultation while the participant was in a seated position. Three blood pressure readings, spaced 1 minute apart, were taken using a sphygmomanometer with an appropriate cuff size. The first reading was discarded, and the last two readings were averaged. If large differences were observed between the second and third readings, an additional reading was taken and the median value of these 3 readings was used.

$d$-ROMs test The d-ROMs values were measured using a kinetic spectrophotometric assay (F.R.E.E System; Diacron, Italy) with intra- and inter-assay coefficients of variation of $2.1 \%$ and $3.1 \%$, respectively. Briefly, a serum sample $(25 \mu \mathrm{L})$ was mixed with an acetic acid buffered solution $(\mathrm{pH} 4.8)$ in a pipette to stabilize the hydrogen ion concentration, and a chromogenic substrate was added to the mixture. In an acidified medium, bivalent and trivalent iron from the protein component of the blood ionizes and works as a catalyst to break down hydroperoxide groups in the blood into alkoxyl and peroxy radicals to become free radicals. The mixture was then incubated in the thermostatic block of the system, and then transferred into a cuvette containing colorless chromogen. The chromogen is oxidized by free radicals to a radical cation with a magenta color. The intensity of the magenta color reflects the concentration of hydroperoxides in the blood, which is proportional to the quantity of ROMs. The magenta color was measured using a photometer (505 nm) after centrifuging for 1 minute to measure the quantity of hydroperoxide. The measurements were expressed in U. Carr. (1 U. Carr. corresponds to $0.08 \mathrm{mg} / \mathrm{dL} \mathrm{H}_{2} \mathrm{O}_{2}$ ).

Statistical analysis: All data are expressed as the mean \pm SD. SPSS 15.0 software (SPSS Inc., Chicago, IL, USA) was used for statistical processing. Student's $t$-test or the chi-square test was performed to determine whether intergroup differences were statistically significant at baseline and during the study. The paired $t$-test was performed to determine whether intragroup differences at baseline and 12 weeks were statistically significant. The relationship between changes in CAVI and other variables were analyzed using simple regression (Pear- 
son's product-moment correlation coefficient: $r$ ). Multivariate logistic regression analysis was used to identify the factors associated with CAVI decrease, and expressed as the odds ratio (OR) with a $95 \%$ confidence interval (CI). In all comparisons, $P$ values less than 0.05 were considered statistically significant.

\section{RESUlTS}

The patient characteristics of the participants in the resveratrol and placebo groups at baseline are shown in Table 1.
Mean BW and BMI were slightly lower in the placebo group than in the resveratrol group. However, the differences were not significant. Among 60 patients who were interviewed, 50 eligible patients with T2DM (HbA1c $>7.0 \%)$ were recruited into the study (Figure). No adverse events due to administration of resveratrol supplement or placebo were observed during the intervention.

Changes in CAVI: CAVI decreased significantly $(P<0.01)$ after 12-week supplementation only in the resveratrol group (Table I). The decrease in CAVI was significantly $(P<0.01)$ greater in the resveratrol group than in the placebo group (Ta-

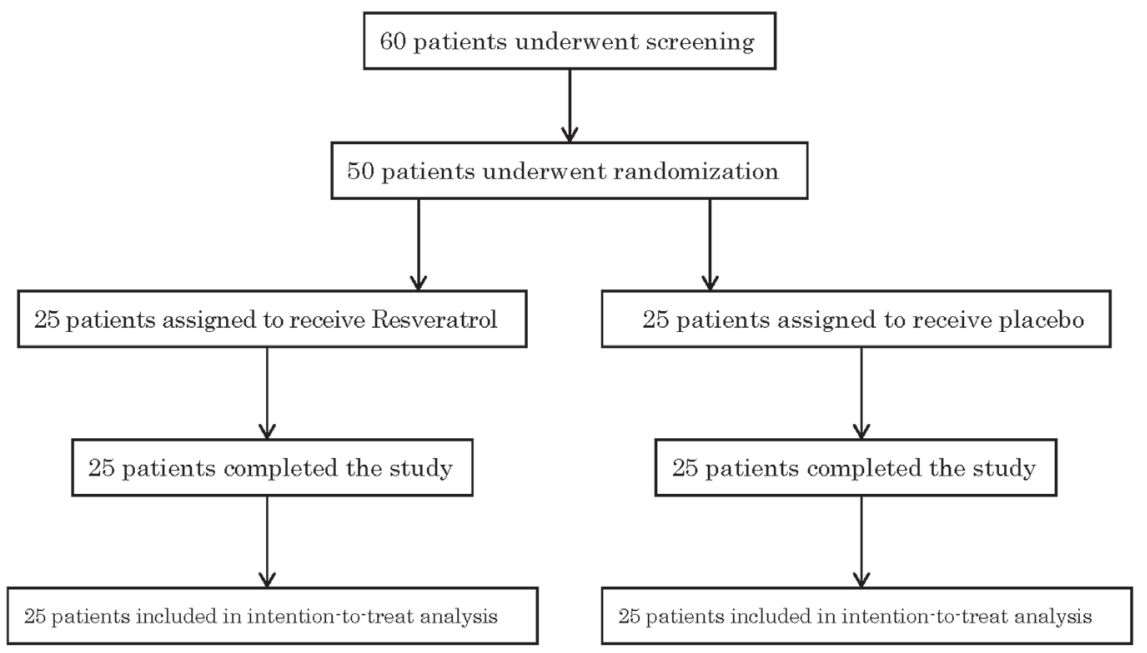

Figure. Patient enrollment flow chart.

Table I. Patient Characteristics in the Resveratrol and Placebo Groups at Baseline and After 12 Weeks of Treatment

\begin{tabular}{lcccc}
\hline & \multicolumn{2}{c}{ Resveratrol } & \multicolumn{2}{c}{ Placebo } \\
& Baseline & 12 weeks & Baseline & 12 weeks \\
\hline$n$ (Male/Female) & $25(15 / 10)$ & - & $25(11 / 14)$ & - \\
Age (years) & $57.4 \pm 10.6$ & - & $58.2 \pm 10.1$ & - \\
Height (cm) & $163.3 \pm 8.8$ & - & $161.6 \pm 10.2$ & - \\
BW (kg) & $69.5 \pm 13.0$ & $68.7 \pm 13.1$ & $63.4 \pm 13.9$ & $63.3 \pm 13.7$ \\
BMI (kg/m) & $26.1 \pm 4.2$ & $25.7 \pm 4.2$ & $24.1 \pm 4.0$ & $24.1 \pm 4.1$ \\
sBP (mmHg) & $137.1 \pm 18.7$ & $131.6 \pm 16.5^{*}$ & $137.1 \pm 25.0$ & $133.2 \pm 26.5$ \\
dBP (mmHg) & $82.0 \pm 9.5$ & $80.5 \pm 11.2$ & $80.8 \pm 11.5$ & $79.9 \pm 11.5$ \\
Hypertension (\%) & $10 / 25(40 \%)$ & - & $9 / 25(36 \%)$ & - \\
FPG (mg/dL) & $152.0 \pm 44.7$ & $157.1 \pm 50.9$ & $138.1 \pm 46.8$ & $143.6 \pm 73.2$ \\
HbAlc (\%) & $7.4 \pm 1.1$ & $7.3 \pm 1.3$ & $7.1 \pm 1.4$ & $7.1 \pm 1.4$ \\
TC (mg/dL) & $187.0 \pm 24.4$ & $189.8 \pm 32.3$ & $205.9 \pm 44.7$ & $207.6 \pm 38.7$ \\
TG (mg/dL) & $123.3 \pm 67.6$ & $126.7 \pm 66.8$ & $139.0 \pm 66.7$ & $136.5 \pm 77.7$ \\
HDL-C (mg/dL) & $56.2 \pm 17.0$ & $58.6 \pm 18.4$ & $56.1 \pm 13.8$ & $56.8 \pm 10.6$ \\
Dyslipidemia (\%) & $8 / 25(32 \%)$ & - & $13 / 25(52 \%)$ & - \\
CAVI & $9.1 \pm 1.1$ & $8.6 \pm 1.0^{* *}$ & $8.9 \pm 1.4$ & $9.0 \pm 1.3$ \\
d-ROMs (U.CARR) & $379.9 \pm 64.4$ & $354.3 \pm 50.6 *$ & $350.4 \pm 72.8$ & $351.5 \pm 79.0$ \\
Medication (\% of patients) & & & & \\
Insulin & 16.0 & - & 8.0 & - \\
Oral hypoglycemic agent & 72.0 & - & 64.0 & - \\
Lipid lowering agent & 40.0 & - & 40.0 & - \\
Calcium channel blocker & 4.0 & - & 16.0 & - \\
ARB/ACE-I & 20.0 & - & 12.0 & - \\
\hline D & & - & & \\
\hline
\end{tabular}

Date are presented as the mean $\pm \mathrm{SD}$, or percent of patients. NS: not significant. $P<0.05,{ }^{* *} P<0.01$ versus baseline, paired $t$-test. BW indicates body weight; BMI, body-mass index; sBP, systolic blood pressure; $\mathrm{dBP}$, diastolic blood pressure; HbAlc, hemoglobin Alc; FPG, fasting plasma glucose; TC, total cholesterol; TG, triglycerides; HDL-C, high density lipoprotein-cholesterol; CAVI, cardio-ankle vascular index; d-ROMs, derivatives of reactive oxygen metabolites; ARB, angiotensin II receptor blocker; and ACE-I, angiotensin enzyme inhibitor. 
ble II).

Changes in glucose and lipid parameters: Fasting plasma glucose, HbA1c, total cholesterol, triglycerides, high-density lipoprotein cholesterol, and LDL-C showed no significant changes during this study in either group (Table I).

Changes in body weight, body mass index, blood pressure, and d-ROMs: $\quad$ BW and BMI decreased slightly after 12 weeks in the resveratrol group (BW: $P=0.083$, BMI: $P=0.092$ ), but did not change significantly in the placebo group. There were no significant differences in the changes of both BW and BMI between the two groups (Table II). Systolic BP decreased significantly after 12 weeks only in the resveratrol group, while diastolic BP showed no significant changes during the study in both groups (Table I). D-ROMs decreased significantly $(P<$ $0.01)$ after 2 weeks only in the resveratrol group (Table I), and the decrease was significantly $(P<0.05)$ greater in the resvera- trol group than in the placebo group (Table II).

Comparison of subgroups classified according to CAVI decrease: We divided all patients into two groups according to the change in CAVI after 12 weeks of supplementation: a responder group with a CAVI decrease greater than 0.5 and a non-responder group with a CAVI decrease of 0.5 or less. Baseline CAVI and the rate of resveratrol administration were higher in the responder group than in the non-responder group (Table III). There were no significant differences in the other parameters between the two groups.

Multivariate logistic regression analysis: Table IV summarizes the results of a multivariate logistic regression analysis for the association between change in CAVI (decrease greater than 0.5 ) and clinical variables. High baseline CAVI and resveratrol administration were identified as significant independent predictors for a CAVI decrease greater than 0.5.

Table II. Comparisons of Changes in Clinical Variables After 12 Weeks of Supplementation Between Resveratrol and Placebo Groups

\begin{tabular}{lccc}
\hline & Resveratrol & Placebo & $P$ \\
\hline$\Delta \mathrm{BW}(\mathrm{kg})$ & $-0.8 \pm 2.1$ & $-0.1 \pm 2.6$ & $\mathrm{NS}$ \\
$\Delta \mathrm{BMI}\left(\mathrm{kg} / \mathrm{m}^{2}\right)$ & $-0.5 \pm 0.8$ & $0.0 \pm 1.0$ & $\mathrm{NS}$ \\
$\Delta \mathrm{sBP}(\mathrm{mmHg})$ & $-5.5 \pm 13.0$ & $-4.0 \pm 19.5$ & $\mathrm{NS}$ \\
$\Delta \mathrm{dBP}(\mathrm{mmHg})$ & $-1.5 \pm 9.3$ & $-0.9 \pm 6.7$ & $\mathrm{NS}$ \\
$\Delta \mathrm{FPG}(\mathrm{mg} / \mathrm{dL})$ & $5.0 \pm 30.7$ & $5.4 \pm 47.7$ & $\mathrm{NS}$ \\
$\Delta \mathrm{HbAlc}(\%)$ & $-0.1 \pm 0.7$ & $0.0 \pm 0.4$ & $\mathrm{NS}$ \\
$\Delta \mathrm{TC}(\mathrm{mg} / \mathrm{dL})$ & $2.7 \pm 24.2$ & $1.7 \pm 38.6$ & $\mathrm{NS}$ \\
$\Delta \mathrm{TG}(\mathrm{mg} / \mathrm{dL})$ & $3.4 \pm 47.2$ & $-2.4 \pm 75.4$ & $\mathrm{NS}$ \\
$\Delta$ HDL-C $(\mathrm{mg} / \mathrm{dL})$ & $2.4 \pm 7.6$ & $0.6 \pm 6.8$ & $\mathrm{NS}$ \\
$\Delta$ CAVI & $-0.4 \pm 0.7$ & $0.1 \pm 0.5$ & $<0.01$ \\
$\Delta \mathrm{d}-\mathrm{ROMs}(\mathrm{U} . \mathrm{CARR})$ & $-25.6 \pm 41.8$ & $1.0 \pm 43.4$ & $<0.05$ \\
\hline
\end{tabular}

Data are presented as the mean \pm SD. NS indicates not significant, unpaired $t$-test. Abbreviations are as in Table I.

Table III. Comparison of Baseline Characteristics in Responder Group and Non-Responder Group Based on Decrease in CAVI

\begin{tabular}{|c|c|c|c|}
\hline & Responder group & Non-responder group & $P$ \\
\hline$n$ (Male/Female) & $15(9 / 6)$ & $35(17 / 18)$ & $\mathrm{NS}^{*}$ \\
\hline Age (years) & $57.0 \pm 11.67$ & $58.17 \pm 9.75$ & $\mathrm{NS}^{*}$ \\
\hline Height $(\mathrm{cm})$ & $162.6 \pm 8.6$ & $162.4 \pm 9.9$ & $\mathrm{NS}^{*}$ \\
\hline BW (kg) & $69.9 \pm 12.8$ & $65.01 \pm 13.9$ & $\mathrm{NS}^{*}$ \\
\hline BMI $\left(\mathrm{kg} / \mathrm{m}^{2}\right)$ & $26.5 \pm 4.6$ & $24.5 \pm 3.9$ & $\mathrm{NS}^{*}$ \\
\hline $\mathrm{sBP}(\mathrm{mmHg})$ & $143.7 \pm 21.2$ & $134.3 \pm 21.8$ & $\mathrm{NS}^{*}$ \\
\hline $\mathrm{dBP}(\mathrm{mmHg})$ & $85.8 \pm 10.6$ & $79.5 \pm 10.0$ & $\mathrm{NS}^{*}$ \\
\hline Hypertension (\%) & $7 / 15(46.7 \%)$ & $3 / 35(8.6 \%)$ & $<0.01^{\dagger}$ \\
\hline FPG (mg/dL) & $153.2 \pm 40.4$ & $141.6 \pm 48.1$ & $\mathrm{NS}^{*}$ \\
\hline $\operatorname{HbA1c}(\%)$ & $7.6 \pm 1.0$ & $7.1 \pm 1.4$ & $\mathrm{NS}^{*}$ \\
\hline $\mathrm{TC}(\mathrm{mg} / \mathrm{dL})$ & $189.8 \pm 27.0$ & $199.3 \pm 40.4$ & $\mathrm{NS}^{*}$ \\
\hline $\mathrm{TG}(\mathrm{mg} / \mathrm{dL})$ & $129.4 \pm 70.7$ & $131.9 \pm 66.3$ & $\mathrm{NS}^{*}$ \\
\hline Dyslipidemia (\%) & $5 / 15(33.3 \%)$ & $3 / 35(8.6 \%)$ & $\mathrm{NS}^{\dagger}$ \\
\hline HDL-C (mg/dL) & $57.8 \pm 19.0$ & $55.4 \pm 13.7$ & $\mathrm{NS}^{*}$ \\
\hline CAVI & $9.6 \pm 1.2$ & $8.7 \pm 1.2$ & $<0.01^{*}$ \\
\hline d-ROMs (U.CARR) & $380.7 \pm 70.3$ & $358.5 \pm 69.2$ & $\mathrm{NS}^{*}$ \\
\hline Resveratrol administration (\%) & 86.7 & 34.3 & $<0.001^{*}$ \\
\hline \multicolumn{4}{|l|}{ Medication (\%) } \\
\hline Insulin & 6.7 & 11.4 & $\mathrm{NS}^{*}$ \\
\hline Oral hypoglycemic agent & 80.0 & 62.9 & $\mathrm{NS}^{*}$ \\
\hline Lipid lowering agent & 33.3 & 42.9 & $\mathrm{NS}^{*}$ \\
\hline Calcium channel blocker & 0 & 14.3 & $\mathrm{NS}^{*}$ \\
\hline ARB/ACE-I & 6.7 & 20.0 & $\mathrm{NS}^{*}$ \\
\hline
\end{tabular}

Date are presented as the mean \pm SD. ${ }^{*}$ by unpaired $t$-test, ${ }^{\dagger}$ by chi-square test, ${ }^{\dagger}$ by Fischer's exact test. NS indicates not significant. Responder group: subjects with $\Delta \mathrm{CAVI}<-0.5$. Non-responder group: subjects with $\Delta \mathrm{CAVI} \geq-0.5$. Abbreviations are as in Table I. 
Table IV. Multivariate Logistic Regression Analysis for the Association of CAVI Decrease Greater Than 0.5 With Clinical Variables

\begin{tabular}{lccc}
\hline Variable & Odds ratio & $95 \%$ confidence interval & $P$ \\
\hline Gender (Male; 1 , Female; 0 ) & 1.24 & $0.216-7.14$ & 0.808 \\
Elderly (Age $\geq 65 ; 1,<65 ; 0)$ & 0.186 & $0.0211-1.63$ & 0.129 \\
High CAVI $(\geq 9.0 ; 1,<9.0 ; 0)$ & 16.5 & $1.91-143$ & 0.0109 \\
Obesity (BMI $\geq 25 ; 1,<25 ; 0)$ & 1.92 & $0.261-14.1$ & 0.522 \\
Hypertension (+; $1,-; 0)$ & 2.09 & $0.328-13.3$ & 0.435 \\
Dyslipidemia $(+; 1,-; 0)$ & 1.37 & $0.218-8.60$ & 0.737 \\
Resveratrol administration $(+; 1,-; 0)$ & 16.4 & $2.18-124$ & 0.00664 \\
\hline AIC $=52.533, P<0.0001$. & &
\end{tabular}

\section{Discussion}

The primary finding in the present study was that oral supplementation of resveratrol for 12 weeks decreased CAVI in patients with T2DM. Many previous studies have demonstrated increased CAVI in atherosclerotic diseases such as acute coronary syndrome ${ }^{14)}$ and stroke, ${ }^{15)}$ and these reports indicate that CAVI reflects organic atherosclerosis. We previously conducted a prospective study that followed 1003 consecutive outpatients ( $92.9 \%$ of 1080 patients screened) with metabolic disorders for $6.7 \pm 1.6$ years. The incidence of newonset cardiovascular events was $8.97 \%$ (90 subjects). We demonstrated that CAVI was a predictor of future cardiovascular events independent of traditional coronary risk factors. ${ }^{16)}$ Apart from organic atherosclerosis, CAVI also reflects vascular dysfunction. CAVI is increased in individuals with risk factors for CVD such as current smoking, ${ }^{17)}$ metabolic syndrome, ${ }^{18)}$ diabetes, ${ }^{19)}$ and dyslipidemia. ${ }^{20)}$ Saiki, et $a l^{21)}$ reported that CAVI increased with an increase in the number of risk factors for metabolic syndrome. Moreover, CAVI has been shown to decrease following various interventions to reduce coronary risks, including some medications for metabolic disorders, ${ }^{22,23)}$ weight reduction, ${ }^{24)}$ and smoking cessation. ${ }^{25)}$ Therefore, CAVI is considered to be a good physiological surrogate marker for vascular injury because it can detect reversible vascular disorders, and is useful to assess the effect of treatments. Several reports have demonstrated the effects of resveratrol on atherosclerosis. Jeon, et $a l^{26)}$ reported that 12-week supplementation of resveratrol suppressed high fat diet-induced lipid accumulation in the aorta with improvement of obesity and dyslipidemia in Apo E knockout mice. Regarding the effect on arterial stiffness, Mattison, et $a l^{27)}$ reported that 2-year supplementation of resveratrol decreased pulse wave velocity in rhesus macaque. These results are consistent with our findings. The present study is the first that assessed the effect of resveratrol on arterial stiffness in humans. Taken together, resveratrol may be useful as an anti-arteriosclerosis therapy in diabetic patients.

Interestingly, slight reductions of body weight $(P=0.083)$ and BMI $(P=0.092)$ were observed in the resveratrol group. Recent studies have shown that resveratrol reduces body fat in animals. Kim, et $\mathrm{al}^{28)}$ reported that resveratrol decreased body weight, visceral fat, and blood levels of triglycerides and cholesterol by suppressing the feeding center and inhibiting adipogenesis. Several studies focused on the potential anti-adipogenic effect of resveratrol under in vitro conditions. Lasa, et $a l^{29)}$ reported that resveratrol increased fatty acid consumption via enhancement of CPT1 and PGC1 $\alpha$ expressions in differentiated 3T3-L1 adipocytes. Furthermore, Wang, et $a l^{30)}$ reported that resveratrol induced brown-like adipocyte formation in white fat tissue. These findings suggest that the observed weight loss induced by resveratrol supplementation in this study may reflect reduction of body adiposity, but the mechanism remains to be studied. These results are valuable because there are few studies that assess the effect of resveratrol on body composition in humans.

D-ROMs, a marker of oxidative stress, decreased only in the resveratrol group. The d-ROMs level is known to be proportional to serum hydroperoxide concentration. In this test, the concentrations of peroxidation products of proteins, peptides, amino acids, lipids, and fatty acids are measured by color reaction. The d-ROMs test comprehensively evaluates the status of oxidative stress level in vivo. ${ }^{31)}$ Blood d-ROMs level increases in diseases with enhanced oxidative stress, and decreases by treatment that reduces oxidative stress. ${ }^{32,33)}$ Regarding the effect of resveratrol on oxidative stress, a report has shown that resveratrol treatment decreases ROS production induced by TNF- $\alpha$ or NF- $\kappa \mathrm{B}$ in human vascular endothelial cells. ${ }^{34)}$ Oxidative stress is known to be elevated in diabetic conditions through the generation of advanced glycation end products (AGEs) and activation of NADPH oxidase. ${ }^{35)}$ We previously reported that glucose-lowering treatment using an oral hypoglycemic agent decreased oxidative stress. ${ }^{36)}$ Taken together, previous findings and the results in this study suggest that resveratrol supplementation may reduce oxidative stress in patients with T2DM. Multivariate regression analysis detected no significant relationship between CAVI decrease and clinical variables other than resveratrol administration and baseline CAVI. However, decreases in serum d-ROMs level and systolic BP and slight weight loss were found only in the resveratrol group. We previously reported that the insulin-sensitizer pioglitazone decreased CAVI accompanied by a decrease in 8 -OhdG. ${ }^{36)}$ We also reported that visceral adiposity was related to CAVI elevation, and weight reduction induced a decrease in CAVI. ${ }^{24)}$ Taken together, reduced oxidative stress and weight loss observed in the present study may be involved in the mechanisms that improve arterial stiffness. On the other hand, other underlying mechanisms also have been suggested. Resveratrol has been reported to improve endothelial dysfunction by increasing NO production. We previously demonstrated a negative correlation between CAVI and flow-mediated dilation, suggesting that CAVI reflects endothelial function. ${ }^{37,38)}$ Therefore, improvement of endothelial function might be potentially another mechanism.

One of the limitations of this study is that the sample size was relatively small and the study period was only 12 weeks. Thus, the long-term effect of resveratrol and outcome are still 
unclear. Another is that we adopted a convenient cut-off value of change in CAVI between the responder and non-responder groups determined to be 0.5 in order to exhibit the efficacy of resveratrol on CAVI. However, none is still established as an actual cut-off value of change in CAVI to prevent CV events. Therefore, further large-scale blind studies with longer followup periods and a cross-over design may be necessary. Despite the limitations, we were able to demonstrate the effects of resveratrol on arterial stiffness and oxidative stress in T2DM patients in this study. From these viewpoints, a large-scale prospective multicenter cohort study including healthy subjects without atherogenic risks is necessary.

In conclusion, this study demonstrated that 12-week oral supplementation of resveratrol improved arterial stiffness and reduced oxidative stress in patients with T2DM. Resveratrol may be beneficial in preventing the development of atherosclerosis induced by diabetes. However, a large-scale cohort study is required to validate the present findings.

\section{REFERENCES}

1. Kannel WB, McGee DL. Diabetes and glucose tolerance as risk factors for cardiovascular disease : the Framingham study. Diabetes Care 1979; 2: 120-6.

2. Haffner SM, Lehto S, Ronnemaa T, Pyörälä K, Laakso M. Mortality from coronary heart disease in subjects with type 2 diabetes and in nondiabetic subjects with and without prior myocardial infarction. N Engl J Med 1998; 339: 229-34.

3. Karabulut A, Iltumur K, Toprak N, et al. Insulin response to oral glucose loading and coronary artery disease in nondiabetics. Int Heart J 2005; 46: 761-70.

4. Baur JA, Pearson KJ, Price NL, et al. Resveratrol improves health and survival of mice on a high-calorie diet. Nature 2006; 444: 337 42.

5. Bonkowski MS, Sinclair DA. Slowing ageing by design: the rise of NAD (+) and sirtuin-activating compounds. Nat Rev Mol Cell Biol 2016; 17: 679-90. (Review)

6. Meng C, Liu JL, Du AL. Cardioprotective effect of resveratrol on atherogenic diet-fed rats. Int J Clin Exp Pathol 2014; 7: 7899-906.

7. Chang GR, Chen PL, Hou PH, Mao FC. Resveratrol protects against diet-induced atherosclerosis by reducing low-density lipoprotein cholesterol and inhibiting inflammation in apolipoprotein E-deficient mice. Iran J Basic Med Sci 2015; 18: 1063-71.

8. Riccioni G, Gammone MA, Tettamanti G, Bergante S, Pluchinotta FR, D'Orazio N. Resveratrol and anti-atherogenic effects. Int J Food Sci Nutr 2015; 66: 603-10. (Review)

9. Phyu HE, Irwin JC, Vella RK, Fenning AS. Resveratrol shows neuronal and vascular-protective effects in older, obese, streptozotocin-induced diabetic rats. Br J Nutr 2016; 115: 1911-8.

10. Shirai K, Utino J, Saiki A, et al. Evaluation of blood pressure control using a new arterial stiffness parameter, cardio-ankle vascular index (CAVI). Curr Hypertens Rev 2013; 9: 66-75.

11. Shirai K, Utino J, Otsuka K, Takata M. A novel blood pressure-independent arterial wall stiffness parameter; cardio-ankle vascular index (CAVI). J Atheroscler Thromb 2006; 13: 101-7.

12. Takaki A, Ogawa H, Wakeyama T, et al. Cardio-ankle vascular index is a new noninvasive parameter of arterial stiffness. Circ J 2007; 71: 1710-4.

13. Nakamura K, Tomaru T, Yamamura S, Miyashita Y, Shirai K, Noike H. Cardio-ankle vascular index is a candidate predictor of coronary atherosclerosis. Circ J 2008; 72: 598-604.

14. Namekata T, Suzuki K, Ishizuka N, Shirai K. Establishing baseline criteria of cardio-ankle vascular index as a new indicator of arteriosclerosis: a cross-sectional study. BMC Cardiovas Disord 2011; $11: 51$

15. Suzuki J, Sakakibara R, Tomaru T, et al. Stroke and cardio-ankle vascular stiffness index. J Stroke Cerebrovasc Dis 2013; 22: 1715 .

16. Sato Y, Nagayama D, Saiki A, et al. Cardio-ankle vascular index is independently associated with future cardiovascular events in outpatients with metabolic disorders. J Atheroscler Thromb 2016; 23 : 596-605.

17. Kubozono T, Miyata M, Uegama K, et al. Acute and chronic effects of smoking on arterial stiffness. Circ J 2011; 95: 698-702.

18. Satoh-Asahara N, Suganami T, Majima T, et al; Japan Obesity and Metabolic Syndrome Study (JOMS) Group. Urinary cystatin C as a potential risk marker for cardiovascular disease and chronic kidney disease in patients with obesity and metabolic syndrome. Clin J Am Soc Nephrol 2011; 6: 265-73

19. Tsuboi A, Ito C, Fujikawa R, Yamamoto H, Kihara Y. Association between the postprandial glucose levels and arterial stiffness measured according to the cardio-ankle vascular index in non-diabetic subjects. Intern Med 2015; 54: 1961-9.

20. Soska V, Dobsak P, Dusek L, et al. Cardio-ankle vascular index in heterozygous familial hypercholesterolemia. J Atheroscler Thromb 2012; 19: 453-61.

21. Saiki A, Sato Y, Watanabe R, et al. The role of a novel arterial stiffness parameter, Cardio-Ankle Vascular Index (CAVI), as a surrogate marker for cardiovascular diseases. J Atheroscler Thromb 2016; 23: 155-68. (Review)

22. Kurisu S, Shimonaga T, Iwasaki T, et al. Effects of ezetimibe on serum polyunsaturated fatty acids in patients with coronary artery disease. Int Heart J 2013; 54: 254-7.

23. Takahashi R, Taguchi N, Suzuki M, et al. Cholesterol and triglyceride concentrations in lipoproteins as related to carotid intimamedia thickness. Int Heart J 2012; 53: 29-34.

24. Nagayama D, Endo K, Ohira M, et al: Effects of body weight reduction on cardio- ankle vascular index (CAVI). Obes Res Clin Pract 2013; 7: e139-e145.

25. Noike H, Nakamura K, Sugiyama Y, et al. Changes in cardio-ankle vascular index in smoking cessation. J Atheroscler Thromb 2010; 17: 517-25.

26. Jeon SM, Lee SA, Choi MS. Antiobesity and vasoprotective effects of resveratrol in apoE-deficient mice. J Med Food 2014; 17: 310-6.

27. Mattison JA, Wang M, Bernier M, et al. Resveratrol prevents high fat/sucrose diet-induced central arterial wall inflammation and stiffening in nonhuman primates. Cell Metab 2014; 20: 183-90.

28. Kim S, Jin Y, Choi Y, Park T. Resveratrol exerts anti-obesity effects via mechanisms involving down-regulation of adipogenic and inflammatory processes in mice. Biochem Pharmacol 2011; 81: 1343-51.

29. Lasa A, Churruca I, Eseberri I, Andrés-Lacueva C, Portillo MP. Delipidating effect of resveratrol metabolites in 3T3-L1 adipocytes. Mol Nutr Food Res 2012; 56: 1559-68.

30. Wang S, Liang X, Yang Q, et al. Resveratrol induces brown-like adipocyte formation in white fat through activation of AMP-activated protein kinase (AMPK) $\alpha 1$. Int J Obes 2015; 39: 967-76.

31. Ebisawa S, Kashima Y, Miyashita Y, et al. Impact of endovascular therapy on oxidative stress in patients with peripheral artery disease. Circ J 2014; 78: 1445-50.

32. Nakajima A, Aoki Y, Shibata Y, et al. Identification of clinical parameters associated with serum oxidative stress in patients with rheumatoid arthritis. Mod Rheumatol 2014; 24: 926-30.

33. Kato T, Umeda A, Miyagawa K, et al. Varenicline-assisted smoking cessation decreases oxidative stress and restores endothelial function. Hypertens Res 2014; 37: 655-8.

34. Gobec M, Tomašič T, Markovič T, Mlinarič-Raščan I, Dolenc MS, Jakopin Ž. Antioxidant and anti-inflammatory properties of 1,2,4-oxadiazole analogs of resveratrol. Chem Biol Interact 2015; 240: 200-7.

35. Matsui T, Nakamura N, Ojima A, Nishino Y, Yamagishi SI. Sulforaphane reduces advanced glycation end products (AGEs)-induced inflammation in endothelial cells and rat aorta. Nutr Metab Cardiovasc Dis 2016; 26: 797-807.

36. Ohira M, Yamaguchi T, Saiki A, et al. Pioglitazone improves the 
cardio-ankle vascular index in patients with type 2 diabetes mellitus treated with metformin. Diabetes Metab Syndr Obes 2014; 7: 313-9.

37. Endo K, Saiki A, Ohira M, Miyashita Y, Shirai K. Cardio-ankle vascular index may reflect endothelial function in type 2 diabetes.
Int J Clin Pract 2011; 65: 1200-1.

38. Hirata Y, Nagata D, Suzuki E, Nishimatsu H, Suzuki J, Nagai R. Diagnosis and treatment of endothelial dysfunction in cardiovascular disease. Int Heart J 2010; 51: 1-6. (Review) 